\title{
Basilar artery thrombus magnetic susceptibility for cardioembolic stroke identification
}

\author{
Jie Chen ${ }^{1 \#}$, Zhe Zhang ${ }^{2 \#}$, Ximing $\mathrm{Nie}^{1 \wedge}$, Yuyuan $\mathrm{Xu}^{2}$, Chunlei Liu ${ }^{3}$, Xingquan Zhao ${ }^{1 \wedge}$, Yongjun Wang ${ }^{1,2}$ \\ ${ }^{1}$ Department of Neurology, Beijing Tiantan Hospital, Capital Medical University, Beijing, China; ${ }^{2}$ China National Clinical Research Center for \\ Neurological Diseases, Beijing, China; ${ }^{3}$ Department of Electrical Engineering and Computer Sciences, University of California, Berkeley, CA, USA
}

\#These authors contributed equally to this work.

Correspondence to: Yongjun Wang. Department of Neurology, Beijing Tiantan Hospital, Capital Medical University, 119 South 4th Ring W Rd, Fengtai District, Beijing 100070, China. Email: yongjunwang@ncrend.org.cn.

\begin{abstract}
The susceptibility vessel sign (SVS) on magnetic resonance imaging (MRI) in the middle cerebral artery is a reliable biomarker for cardioembolic ischemic stroke. As for the basilar artery (BA), the magnetic susceptibility-related image signs, the quantitative evaluation of the corresponding thrombus, and their prediction of cardioembolism require further study. This study aimed to assess thrombus susceptibilityrelated image characteristics using susceptibility-weighted imaging (SWI) and quantitative susceptibility mapping (QSM) MRI in acute BA occlusion and analyze their associations with cardioembolic ischemic stroke. A total of 41 consecutive acute ischemic stroke patients with BA occlusion within 48 h of onset were recruited. A three-dimensional (3D) multi-echo scan on a 3.0T MRI scanner was applied to all participants, and the quantitative susceptibility value of the BA thrombus was computed using QSM. The presence and diameter of SWI SVS in patients with cardioembolic stroke were not significantly different from those with other etiologies. While BA thrombus's susceptibility in patients with cardioembolic stroke was significantly higher than that in patients with non-cardioembolic stroke $(0.42 \pm 0.09$ vs. $0.22 \pm 0.11 \mathrm{ppm}, \mathrm{P}<0.001)$. Receiver operating characteristic (ROC) curve analysis showed that the optimal cutoff value for the QSM susceptibility of BA thrombus in the identification of cardioembolic stroke was $0.33 \mathrm{ppm}$, with sensitivity, specificity, positive predictive value, and negative predictive value of $81.8 \%$ (95\% CI: $48.2 \%$ to $97.7 \%$ ), 81.3\% (95\% CI: $63.6 \%$ to $92.8 \%$ ), $60.0 \%$ (95\% CI: $40.9 \%$ to $76.5 \%$ ), and $92.9 \%$ (95\% CI: $78.6 \%$ to $97.9 \%$ ), respectively. Magnetic susceptibility in BA thrombus could discriminate cardiogenic embolism from other stroke subtypes, thus becoming a novel biomarker of cardiogenic stroke in acute BA occlusion.
\end{abstract}

Keywords: Susceptibility vessel sign (SVS); quantitative susceptibility mapping (QSM); basilar artery (BA) occlusion; cardioembolic stroke

Submitted May 20, 2021. Accepted for publication Sep 01, 2021.

doi: 10.21037/qims-21-547

View this article at: https://dx.doi.org/10.21037/qims-21-547

^ ORCID: Jie Chen, 0000-0002-4008-8970; Zhe Zhang, 0000-0001-8220-8443; Ximing Nie, 0000-0002-8380-4076; Xingquan Zhao, 00000001-8345-5147. 


\section{Introduction}

Posterior circulatory ischemic stroke accounts for approximately one-fifth of acute ischemic strokes and has a high risk of early recurrence (1). Therefore, early stroke etiology diagnosis and effective secondary prevention are essential for prognosis.

Cardioembolic sources are a common but sometimes undetected cause. Recent studies have demonstrated that the susceptibility vessel sign (SVS) on magnetic resonance imaging (MRI) in the middle cerebral artery is a reliable biomarker for cardioembolic ischemic stroke (2-4). However, most SVS studies were performed in the middle cerebral arteries, which were defined as a hypointense signal on $\mathrm{T}^{*}$-weighted or susceptibility-weighted imaging (SWI), and the SVS diameter should exceed the contralateral artery (2-4). It is challenging for the basilar artery (BA) to determine whether the hypointense signal is a blooming artifact due to the lack of contralateral artery reference and the varying luminal diameter of the BA with deviation vertebrobasilar dolichoectasia (5).

Quantitative susceptibility mapping (QSM) is a novel technique determining each voxel's magnetic susceptibility based on gradient-recalled echo (GRE) phase images $(6,7)$. The hypointense signal of the SVS in SWI is often the result of an increase in local susceptibility. We postulated that QSM could provide a quantitative description of the paramagnetic contents in the BA thrombus and thus reflect the source of the thrombus. We assessed the thrombus susceptibility on QSM in acute BA occlusion and analyzed its association with cardioembolic ischemic stroke to test this hypothesis.

We present the following article in accordance with the Standards for Reporting Diagnostic accuracy studies (STARD) reporting checklist (available at https://dx.doi. org/10.21037/qims-21-547).

\section{Methods}

\section{Study participants}

In this prospective observational study, we consecutively enrolled patients between November 2018 and February 2020 from Beijing Tiantan Hospital, who were aged $\geq 18$ years with acute ischemic stroke caused by basilar arterial occlusion confirmed by magnetic resonance angiography (MRA) performed within $48 \mathrm{~h}$ of stroke onset.

This study was conducted in accordance with the Declaration of Helsinki (as revised in 2013) and approved by the Research Ethics Committee of Beijing Tiantan Hospital. All participants or their guardians provided written informed consent.

\section{Image analysis}

All MRIs were obtained using a 3T MRI scanner (Ingenia; Philips, Best, The Netherlands). The QSM imaging protocol was a three-dimensional (3D) multi-echo gradientecho sequence with first echo time $=6 \mathrm{~ms}$, echo spacing $=6.7 \mathrm{~ms}$, field of view $=230 \times 230 \times 140 \mathrm{~mm}^{3}$, and voxel size $=0.48 \times 0.48 \times 0.75 \mathrm{~mm}^{3}$. The multi-echo modulus and phase images were stored for quantitative susceptibility calculation.

All images were saved in the nifti format (https:// nifti.nimh.nih.gov/nifti-1/) for quantitative susceptibility calculation and analysis of the region of interest (ROI). The QSM [with the unit of parts per million (ppm)] was performed using the STI Suite toolbox (v2.2) in MATLAB (Mathworks, Natick, MA, USA) (6), and then loaded in 3D Slicer (8) (https://www.slicer.org/) for ROI analysis. For each participant, the thrombus ROI was manually drawn on the hyperintense region on 1 central axial slice in the BA of the QSM image. As the QSM provides relative rather than absolute values of magnetic susceptibility, the susceptibility of thrombi was normalized using the white matter reference (9). The reference ROI was drawn on one axial slice in the normal-appearing white matter in bilateral centrum semioval (Figure S1). The thrombus susceptibility value for each participant was recorded as the difference between the mean susceptibility values of the thrombus ROI and the reference ROI. The ROI drawing and QSM quantification output were performed by 2 investigators (J.C. and Z.Z.) who were blinded to the clinical data (10).

The SWI was generated from gradient-echo data using the vendor-provided SWIp sequence. The SVS was defined as in previous research on SWI by hypointense signs in the corresponding symptomatic occlusive vessels (2). The diameter of the SVS-related hypointense signal was measured on SWI, using the maximum radial distance of the hypointense vessel (4). The SWI analysis was performed on an image viewer (RadiAnt DICOM viewer, https://www. radiantviewer.com/).

\section{Clinical assessments}

To increase cardioembolic stroke detection, all recruited patients underwent a 7-day electrocardiograph monitoring, 


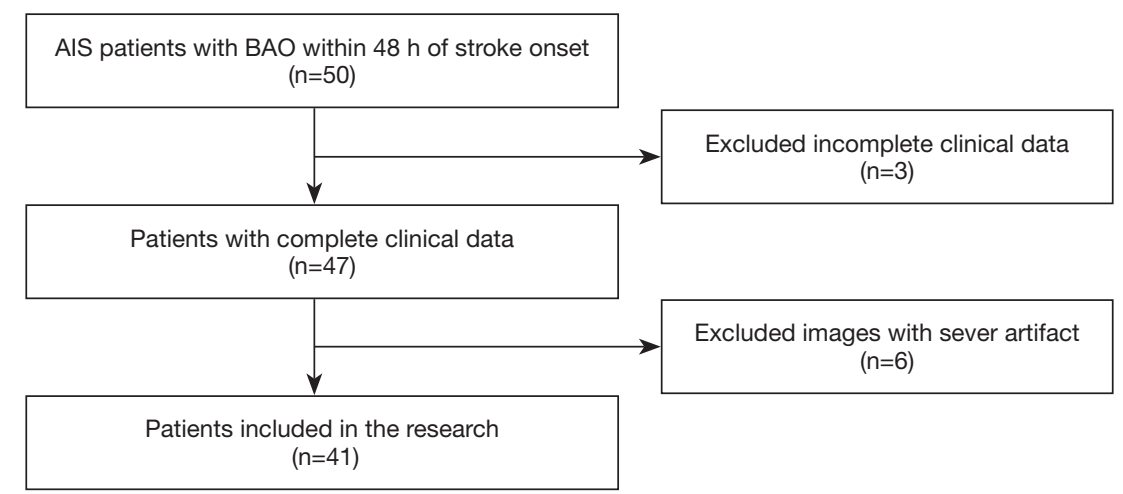

Figure 1 Flow chart of study. AIS, acute ischemic stroke; BAO, basilar artery occlusion.

transthoracic echocardiography, and serum B-type natriuretic peptide test. The ischemic stroke subtype was determined by 2 experienced neurologists (X.N. and Y.X.) following the Trial of ORG 10172 in Acute Stroke Treatment (TOAST) (11).

\section{Statistical analysis}

Participants classified as having a cardioembolic stroke due to BA occlusion were compared with other stroke subtypes. Baseline characteristics of both groups were compared using the Mann-Whitney U test or Student's $t$-test for continuous variables and Pearson's $\chi^{2}$ test for categorical variables. Mann-Whitney U test or Student's $t$-test were used for continuous variable comparison of baseline characteristics, and Pearson's $\chi^{2}$ test was used for categorical variables comparison. The intraclass correlation coefficient (ICC) was performed to measure the interobserver agreement of thrombus susceptibility and SVS diameter, and receiver operating characteristic (ROC) analysis to determine the optimal cutoff value for identifying cardioembolic stroke. All statistical significance levels were set at $\mathrm{P}<0.05$. The ROC analysis was performed using MedCalc V.19.1 (MedCalc Software, Ostend, Belgium), and other statistical analyses were performed using SPSS Version 24 (IBM Corp., Chicago, IL, USA).

\section{Results}

We consecutively enrolled 50 patients with acute BA occlusion between November 2018 and February 2020, although 9 patients were excluded because of inadequate clinical data and severe image artifacts (Figure 1). A total of 41 patients were included in the present study, 17 women $(41.5 \%)$ and 24 men $(58.5 \%)$, with a mean age of $64.24 \pm 11.56$ years and a median National Institutes of Health Stroke Scale (NIHSS) of 24 (interquartile range, 13-36). All participants underwent MRI scans within $48 \mathrm{~h}$ from stroke onset, with a mean time of $17.80 \pm 14.60 \mathrm{~h}$. Regarding the ischemic stroke subtype, 11 (26.8\%) participants were determined with cardioembolism, and 30 participants with non-cardioembolism, including 20 large-artery atherosclerosis (48.8\%), 3 dissection (4.5\%), and 6undetermined etiology $(14.6 \%)$. The baseline characteristics and factors associated with cardioembolic stroke are shown in Table 1.

The hypointense signal on SWI in the corresponding BA occlusion was present in 35 (85.4\%) participants, the diameter of which in the cardioembolic group $(4.32 \pm 1.23 \mathrm{~mm})$ was larger than that in the non-cardioembolic group $(3.56 \pm 1.15 \mathrm{~mm})$, but without statistical significance $(\mathrm{P}=0.084)$. The ICC of the SVS diameter was 0.731 .

The susceptibility of the hyperintense signal at BA (corresponding to the SWI hypointense signal) on QSM is also described in Figure 2. The interobserver agreement of the mean thrombus susceptibility measured by the intraclass correlation coefficient was 0.966 . The BA thrombus's susceptibility in participants with cardioembolic stroke was significantly higher than that in those with non-cardioembolic stroke $(0.42 \pm 0.09$ vs. $0.22 \pm 0.11 \mathrm{ppm}$, $\mathrm{P}<0.001)$. The ROC curve analysis showed that the area under the curve (AUC) of thrombus susceptibility was 0.89 (95\% CI: 0.80 to $0.99, \mathrm{P}<0.0001$ ). The optimal cutoff value for the susceptibility of BA thrombus on QSM while identifying a cardioembolic stroke was $0.33 \mathrm{ppm}$ (Figure 3). Furthermore, the QSM susceptibility $(\geq 0.33 \mathrm{ppm})$ yielded a sensitivity, specificity, positive predictive value, and negative predictive value for the cardioembolic stroke of $81.8 \%$ (95\% CI: $48.2 \%$ to $97.7 \%$ ), 
Table 1 Baseline characteristics

\begin{tabular}{lccc}
\hline & CE $(\mathrm{n}=11)$ & Non-CE $(\mathrm{n}=30)$ & $\mathrm{P}$ value \\
\hline Age, mean \pm SD & $66.18 \pm 9.94$ & $63.53 \pm 12.18$ & 0.522 \\
Gender, male (\%) & $6(54.5)$ & $18(60.0)$ & 0.753 \\
NIHSS, median (IQR) & $31(13,41)$ & $22(12,31)$ & 0.329 \\
Atrial fibrillation, $\mathrm{n}(\%)$ & $10(90.1)$ & $23(76.7)$ & $<0.001$ \\
Hypertension, $\mathrm{n}(\%)$ & $9(81.8)$ & $7(23.3)$ & 0.724 \\
Diabetes, $\mathrm{n}(\%)$ & $5(45.5)$ & $1(3.3)$ & 0.168 \\
Intravenous alteplase, $\mathrm{n}(\%)$ & $2(18.2)$ & $18.94 \pm 15.85$ & 0.106 \\
Time to MRI, h, mean \pm SD & $14.69 \pm 10.43$ & 0.415 \\
\hline
\end{tabular}

CE, cardioembolism; MRI, magnetic resonance imaging; NIHSS, National Institutes of Health Stroke Scale; non-CE, non-cardioembolism; $\mathrm{SD}$, standard deviation.
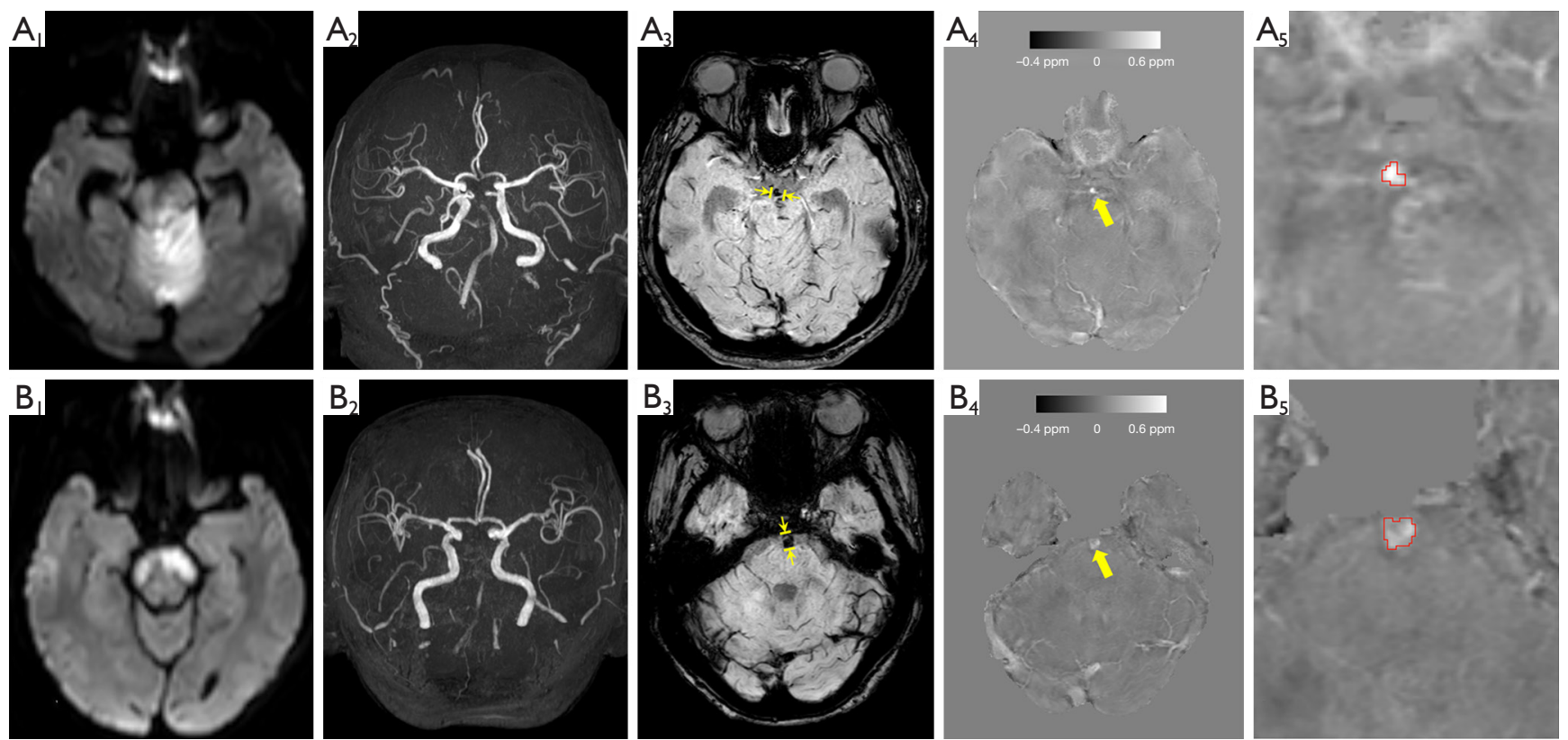

Figure 2 Illustration image for QSM measurement. (A) A patient with cardioembolic stroke (58 years old, male) who presents with acute cerebellum and brainstem infarctions on DWI, image captured $9.5 \mathrm{~h}$ following the onset of coma (A1), basilar artery occlusion on MRA (A2), and hypointense signal on SWI with the diameter of $4.16 \mathrm{~mm}$ in the occluded artery (A3, yellow arrow). QSM shows corresponding hyperintense signals in the occluded basilar artery (A4, yellow arrowhead). The ROI is drawn in axial view at the central slice of the thrombus, with a mean susceptibility value of $0.351 \mathrm{ppm}$ (A5). (B) A patient presented with right hemiparesis and aphasia (89 years old, female); and the stroke subtype was large artery atherosclerosis. There were brainstem infarctions on DWI (B1), basilar artery occlusion on MRA (B2) and hypointense signal on SWI with the diameter of $4.67 \mathrm{~mm}$ (B3, yellow arrow). The signal in the thrombus on QSM maps was relatively low (B4, yellow arrowhead) with the measured mean susceptibility of 0.208 ppm in the ROI (B5, in red contour). DWI, diffusionweighted imaging; MRA, magnetic resonance angiography; SWI, susceptibility-weighted imaging; QSM, quantitative susceptibility mapping; ROI, region of interest. 


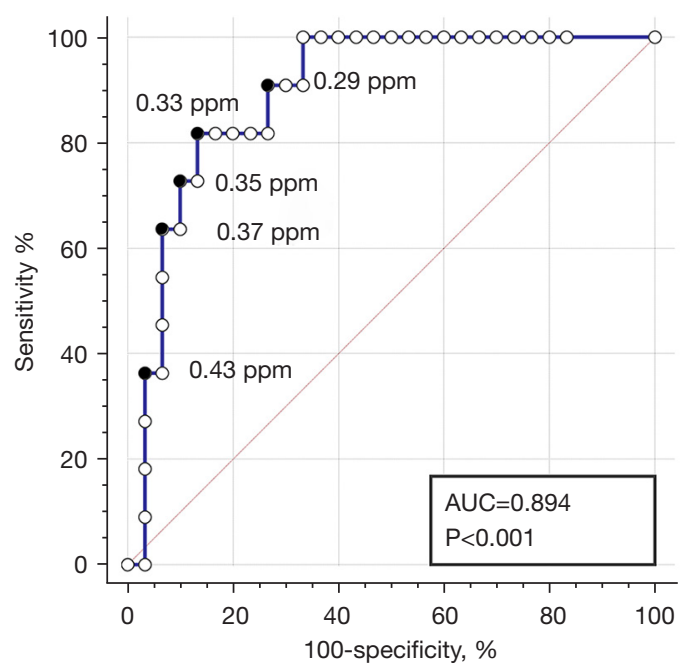

Figure 3 ROC analysis of thrombus susceptibility for predicting cardioembolic stroke. AUC, area under the curve; ROC, receiver operating characteristic.

$81.3 \%$ (95\% CI: $63.6 \%$ to $92.8 \%$ ), $60.0 \%$ (95\% CI: $40.9 \%$ to $76.5 \%$ ), and $92.9 \%$ (95\% CI: $78.6 \%$ to $97.9 \%$ ), respectively.

\section{Discussion}

In the present study, we applied QSM, a novel technique for quantifying thrombi's magnetic susceptibility. We demonstrated the potential of QSM MRI in discriminating cardiogenic thrombi from other stroke subtypes due to acute BA occlusion by assessing the BA thrombus susceptibility in patients with acute ischemic stroke.

The paramagnetic property of deoxyhemoglobin in a thrombus may enhance the blooming hypointense signals, known as SVS, on GRE/SWI sequences. This has been demonstrated to be valuable for predicting stroke subtype and vessel recanalization in anterior circulation stroke (2-4). To our knowledge, only 1 study previously utilized $\mathrm{T} 2{ }^{*} \mathrm{~W}$ GRE to identify thrombi in patients with posterior circulation large artery occlusion (including BA, vertebral artery, and posterior cerebral artery), which reported that SVS was more frequently present in cardioembolic stroke (12). In our study, the SVS diameter metric from SWI did not show a significant difference between the cardioembolic and the non-cardioembolic group, while the quantitative evaluation by QSM did. This result may reveal that quantitative evaluation by QSM demonstrated a more informative assessment of cardiogenic thrombus in the BA region than SWI.

As for the imaging method, since the thrombi in intracranial vessels are usually small in size, the acquisition resolution should be closely monitored. Large imaging voxel size, especially when voxel larger than the thrombus or lumen size, may introduce partial volume effect and decrease the deoxyhemoglobin contrast, resulting in the drop in susceptibility measurement. Thus, high-resolution acquisition, at least 2 times higher resolution compared to lumen and thrombus size for both in-plane and throughplane, is preferred to avoid the potential bias for intracranial thrombus QSM. New imaging techniques and ultra-highfield MRI enable high-resolution QSM and provide new opportunities for future QSM studies.

There were several limitations in this study. Firstly, this study is a single-center study with limited sample size. Although the stroke patients of different subtypes shared similar baseline characteristics, the imbalance may not be ruled out. Secondly, there was no follow-up data to show the predictive value of susceptibility to clinical outcome. Further studies are needed to explore the relationship between susceptibility with recanalization and functional outcome.

Acute BA occlusion is a life-threatening emergency; patients may suffer from sudden and dramatic neurological impairment if not treated promptly and effectively. Like anterior circulation stroke, cardiogenic embolism is the leading cause of acute BA occlusion (5). Our study demonstrated that increased magnetic susceptibility is a novel neuroimaging biomarker for cardiogenic thrombosis, which may help identify patients with cardiogenic embolism for targeted anticoagulant therapy.

\section{Conclusions}

Magnetic susceptibility in BA thrombus could help discriminate cardiogenic embolism from other stroke subtypes, thus becoming a novel biomarker of cardiogenetic stroke in acute BA occlusion.

\section{Acknowledgments}

Funding: This study was supported by the National Natural Science Foundation of China (81701139).

\section{Footnote}

Reporting Checklist: The authors have completed the 
STARD reporting checklist. Available at https://dx.doi. org/10.21037/qims-21-547

Conflicts of Interest: All authors have completed the ICMJE uniform disclosure form (available at https://dx.doi. org/10.21037/qims-21-547). The authors have no conflicts of interest to declare.

Ethical Statement: The authors are accountable for all aspects of the work in ensuring that questions related to the accuracy or integrity of any part of the work are appropriately investigated and resolved. The study was conducted in accordance with the Declaration of Helsinki (as revised in 2013). This study was approved by the Research Ethics Committee of Beijing Tiantan Hospital, and written informed consent was provided by all of the participants or their guardians.

Open Access Statement: This is an Open Access article distributed in accordance with the Creative Commons Attribution-NonCommercial-NoDerivs 4.0 International License (CC BY-NC-ND 4.0), which permits the noncommercial replication and distribution of the article with the strict proviso that no changes or edits are made and the original work is properly cited (including links to both the formal publication through the relevant DOI and the license). See: https://creativecommons.org/licenses/by-nc-nd/4.0/.

\section{References}

1. Markus HS, van der Worp HB, Rothwell PM. Posterior circulation ischaemic stroke and transient ischaemic attack: diagnosis, investigation, and secondary prevention. Lancet Neurol 2013;12:989-98.

2. Cho KH, Kim JS, Kwon SU, Cho AH, Kang DW. Significance of susceptibility vessel sign on T2*-weighted gradient echo imaging for identification of stroke subtypes. Stroke 2005;36:2379-83.

3. Liu M, Li L, Li G. The different clinical value of susceptibility vessel sign in acute ischemic stroke patients under different interventional therapy: A systematic review and meta-analysis. J Clin Neurosci 2019;62:72-9.

4. Kang DW, Jeong HG, Kim DY, Yang W, Lee SH. Prediction of Stroke Subtype and Recanalization Using Susceptibility Vessel Sign on Susceptibility-Weighted Magnetic Resonance Imaging. Stroke 2017;48:1554-9.

5. Mattle HP, Arnold M, Lindsberg PJ, Schonewille WJ, Schroth G. Basilar artery occlusion. Lancet Neurol 2011;10:1002-14.

6. Liu C, Li W, Tong KA, Yeom KW, Kuzminski S. Susceptibility-weighted imaging and quantitative susceptibility mapping in the brain. J Magn Reson Imaging 2015;42:23-41.

7. Kaunzner UW, Kang Y, Zhang S, Morris E, Yao Y, Pandya S, Hurtado Rua SM, Park C, Gillen KM, Nguyen TD, Wang Y, Pitt D, Gauthier SA. Quantitative susceptibility mapping identifies inflammation in a subset of chronic multiple sclerosis lesions. Brain 2019;142:133-45.

8. Fedorov A, Beichel R, Kalpathy-Cramer J, Finet J, FillionRobin JC, Pujol S, Bauer C, Jennings D, Fennessy F, Sonka M, Buatti J, Aylward S, Miller JV, Pieper S, Kikinis R. 3D Slicer as an image computing platform for the Quantitative Imaging Network. Magn Reson Imaging 2012;30:1323-41.

9. Straub S, Schneider TM, Emmerich J, Freitag MT, Ziener CH, Schlemmer HP, Ladd ME, Laun FB. Suitable reference tissues for quantitative susceptibility mapping of the brain. Magn Reson Med 2017;78:204-14.

10. Zheng MZ, Yang QY, Lu XD, Hu SL, Chai C, Shen W, Chang BG, Wang ZY, Xia S. Middle cerebral artery thrombus susceptibility-weighted imaging mapping predicts prognosis. Quant Imaging Med Surg 2019;9:1556-65.

11. Adams HP Jr, Bendixen BH, Kappelle LJ, Biller J, Love BB, Gordon DL, Marsh EE 3rd. Classification of subtype of acute ischemic stroke. Definitions for use in a multicenter clinical trial. TOAST. Trial of Org 10172 in Acute Stroke Treatment. Stroke 1993;24:35-41.

12. Wang N, Hu Q, Wang Q, Zhao J, Dedhia N, Caplan LR. Intracranial Posterior Circulation Large Artery Thrombi Visualized Using Susceptibility-Weighted MRI. J Neuroimaging 2017;27:505-10.
Cite this article as: Chen $\mathrm{J}$, Zhang Z, Nie X, Xu Y, Liu C, Zhao X, Wang Y. Basilar artery thrombus magnetic susceptibility for cardioembolic stroke identification. Quant Imaging Med Surg 2022;12(2):1579-1584. doi: 10.21037/qims21-547 


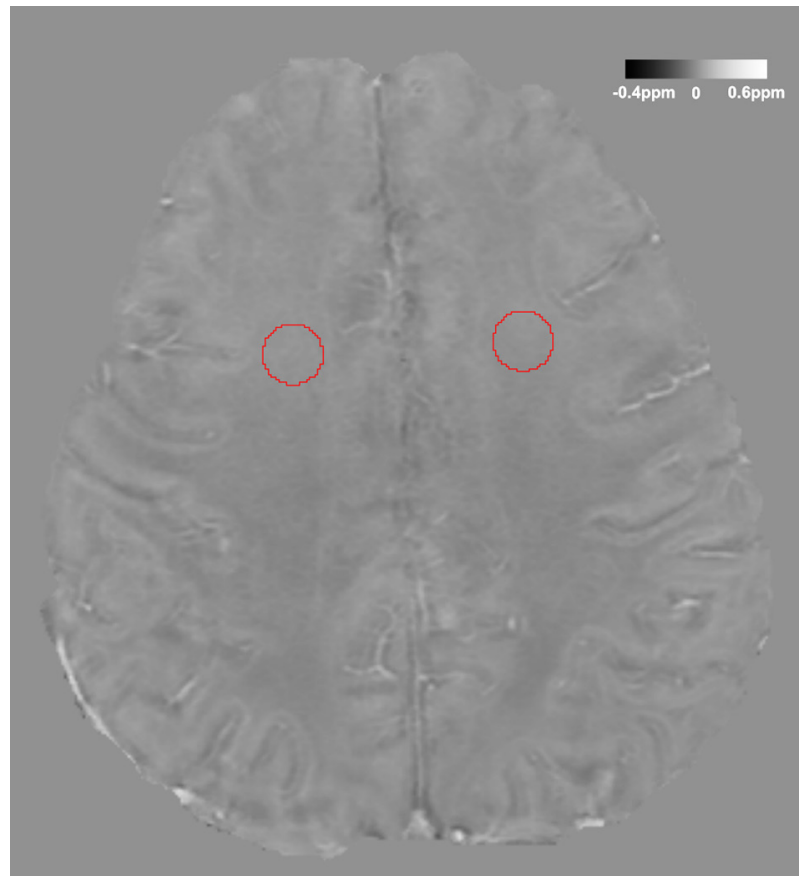

Figure S1 One example of the reference ROI drawing for QSM calculation. For each subject, the reference ROI was drawn on one axial slice in the normal-appearing white matter in bilateral centrum semioval with a diameter of $5 \%$ of the imaging FOV $(=11.5 \mathrm{~mm}=5 \% \times 230 \mathrm{~mm})$. The mean \pm standard deviation susceptibility of all subjects of the reference ROI was $-0.0012 \pm 0.0143 \mathrm{ppm}$. 